\title{
EL PAISAJE VEGETAL HOLOCENO EN EL VALLE ALTO DEL RÍO LOZOYA (PINILLA DEL VALLE, MADRID)
}

Trabajo presentado a las

XXII Jornadas de la Sociedad Española

de Paleontología.

Universidad de León

León, 28-30 de Septiembre de 2006

\author{
M. Blanca RUIZ ZAPATA ${ }^{1}$, Clemencia GÓMEZ \\ GONZÁLEZ1, María José GIL GARCÍA' ${ }^{1}$ José An- \\ tonio LÓPEZ-SÁEZ², Alfredo PÉREZ-GONZÁLEZ', \\ Enrique BAQUEDANO ${ }^{4}$ Juan Luis ARSUAGA
}

\footnotetext{
${ }^{1}$ Departamento de Geología. Edificio de Ciencias. Universidad de Alcalá. 28871 Alcalá de Henares. blanca.ruiz@uah.es

${ }^{2}$ Laboratorio de Arqueobotánica. Departamento de Prehistoria, Instituto de Historia, CSIC, Duque de Medinaceli, 6. 28014 Madrid. alopez@ih.csic.es ${ }^{3}$ Departamento de Geodinámica. Facultad de Geología. Universidad Complutense de Madrid. Ciudad Universitaria s/n. 28040 Madrid. alfredog@geo.ucm.es ${ }^{4}$ Museo Arqueológico Regional. Plaza de Las Bernardas s/n. 28801 Alcalá de Henares. enrique.baquedano@madrid.org

${ }^{5}$ Centro (UCM-ISCIII) de Evolución y Comportamiento Humano. Instituto de Salud Carlos III. C/ Sinesio Delgado, 6, Pabellón 14. 28029 Madrid. jlarsuaga@isciii.es
}

Ruiz Zapata, B., Gómez González, C., Gil García, M. J., López Sáez, J. A., Pérez-González, A., Baquedano, E. \& Arsuaga, J. L. 2007. El paisaje vegetal Holoceno en el valle alto del Lozoya (Pinilla del Valle, Madrid). [The Holocene landscape in the Lozoya upper valley (Pinilla del Valle, Madrid).] Revista Española de Paleontología, 22 (1), 89-94. ISSN 0213-6937.

\begin{abstract}
Pollen and non-pollen palynomorphs analysis of the Buena Pinta cave in the Pinilla del Valle (Madrid) archaeopalaeontological site has been conducted. The results shows a vegetation dynamics from the pollen diagrams can be correlated with the vegetation progressive evolution of an open landscape as adaptation to the installation of hot and dry character climatic conditions. The radiocarbon dating of ${ }^{14} \mathrm{C}$ locates its development, before 57405600 cal BP.
\end{abstract}

Key words: Pinilla del Valle, Lozoya Valley, Buena Pinta cave, pollen, palynomorphs, Holocen, paleoenvironmental.

\section{RESUMEN}

Se ha estudiado el contenido en palinomorfos polínicos y no polínicos, en la secuencia Holocena de la cueva de la Buena Pinta (Yacimiento arqueopaleontológico de Pinilla del Valle, Madrid) . Los resultados obtenidos han puesto de manifiesto una dinámica de la vegetación reflejada en el tránsito progresivo hacia la instalación de un paisaje abierto, como adaptación a unas condiciones climáticas de carácter calido y seco. Las dataciones radiométricas de ${ }^{14} \mathrm{C}$ sitúan, su desarrollo, con anterioridad a 5740-5600 cal BP.

Palabras clave: Pinilla del Valle, valle del Lozoya, cueva de la Buena Pinta, polen, palinomorfos, Holoceno, paleoambiente.

\section{INTRODUCCIÓN}

Los yacimientos arqueopaleontológicos de Pinilla del Valle (Madrid), se localizan a $1.114 \mathrm{~m}$ de altitud en el denominado Calvero de la Higuera (margen derecha de la presa que existe en la cabecera del río Lozoya), frente a la localidad de la que toma el nombre (Fig.1), dentro del sector central de la Sierra de Guadarrama (sector oriental del Sistema Central).

Desde el punto de vista de la vegetación y de acuerdo a las características de zonación biogeográfica (Peinado Lorca \& Rivas-Martínez, 1987), la zona de estudio se 
encuentra enmarcada en el distrito Guadarramense y piso bioclimático Supramediterráneo medio. La temperatura media anual está entre $8^{\circ}$ y $13^{\circ} \mathrm{C}$, la media de las mínimas del mes más frío entre $-4^{\circ}$ y $-1^{\circ} \mathrm{C}$, y la media de las máximas del mes mas frío entre $2^{\circ}$ y $9^{\circ} \mathrm{C}$. El índice de termicidad está entre 60 y 120 (Peinado Lorca \& RivasMartínez, 1987). En el mapa de series de vegetación del Valle del Paular, en la zona central del Valle del Lozoya, el área de estudio se localiza dentro de la serie Luzulo forsteri-Querceto pyrenaicae correspondiente a bosques climatófilos de roble melojo (Quercus pyrenaica) que se desarrollan sobre suelos silíceos en bioclimas supramediterráneos continentalizados y principalmente subhúmedos (Fernández, 1988).

Su génesis está relacionada con la formación de fenómenos kársticos (lapiaces y dolinas en el exterior y cavidades o galerías, en el interior), desarrollados en una serie carbonatada de unos $40 \mathrm{~m}$ de espesor y en cierta medida con los procesos de disección fluvial del arroyo Lontanares y Navalmaillo que confluyen en el río Lozoya. Esta serie carbonatada-detrítica de edad cretácica, presenta un relieve estructural de cuesta, con un buzamiento al $\mathrm{N}$ y descansa sobre rocas graníticas y metamórficas de edad paleozoica; en discordancia erosiva reposan sedimentos terciarios continentales. Los sedimentos cuaternarios, con gran re- presentación en el valle, corresponden a depósitos fluviales, de bloques, gravas, arenas y limo-arcillas de abanicos aluviales y terrazas y a una sedimentación intrakárstica, dando como resultado el relleno de los abrigos y cuevas en el Calvero de la Higuera.

El yacimiento de Pinilla del Valle fue descubierto en 1979 por el equipo de Paleontología de Vertebrados de la Universidad Complutense y excavado de modo sistemático en 1981, bajo la dirección de F. Alférez. Presenta una de las asociaciones paleontológicas más completas del Pleistoceno superior (Alférez et al., 1982, 1992; Alférez, 1985) de la Península Ibérica, a ello se une la presencia de industria lítica y las evidencias de intervención humana, anterior a nuestra especie, puestas de manifiesto en la existencia dos molares de Homo neanderthalensis. En el 2002 se retoman las excavaciones por un equipo interdisciplinar, fruto de las cuales es el descubrimiento de dos nuevos yacimientos: Navalmaillo y la Cueva de la Buena Pinta junto al clásico, denominado actualmente sector Camino (Baquedano et al., 2004; Márquez et al., 2004) y se inician, en los tres yacimientos, los estudios de carácter palinológico (Ruiz Zapata, 2004).

La Cueva de la Buena Pinta, descubierta en 2003, presenta niveles holocenos y pleistocenos, estos últimos con fauna e industria lítica; en la campaña de 2004 se mues-

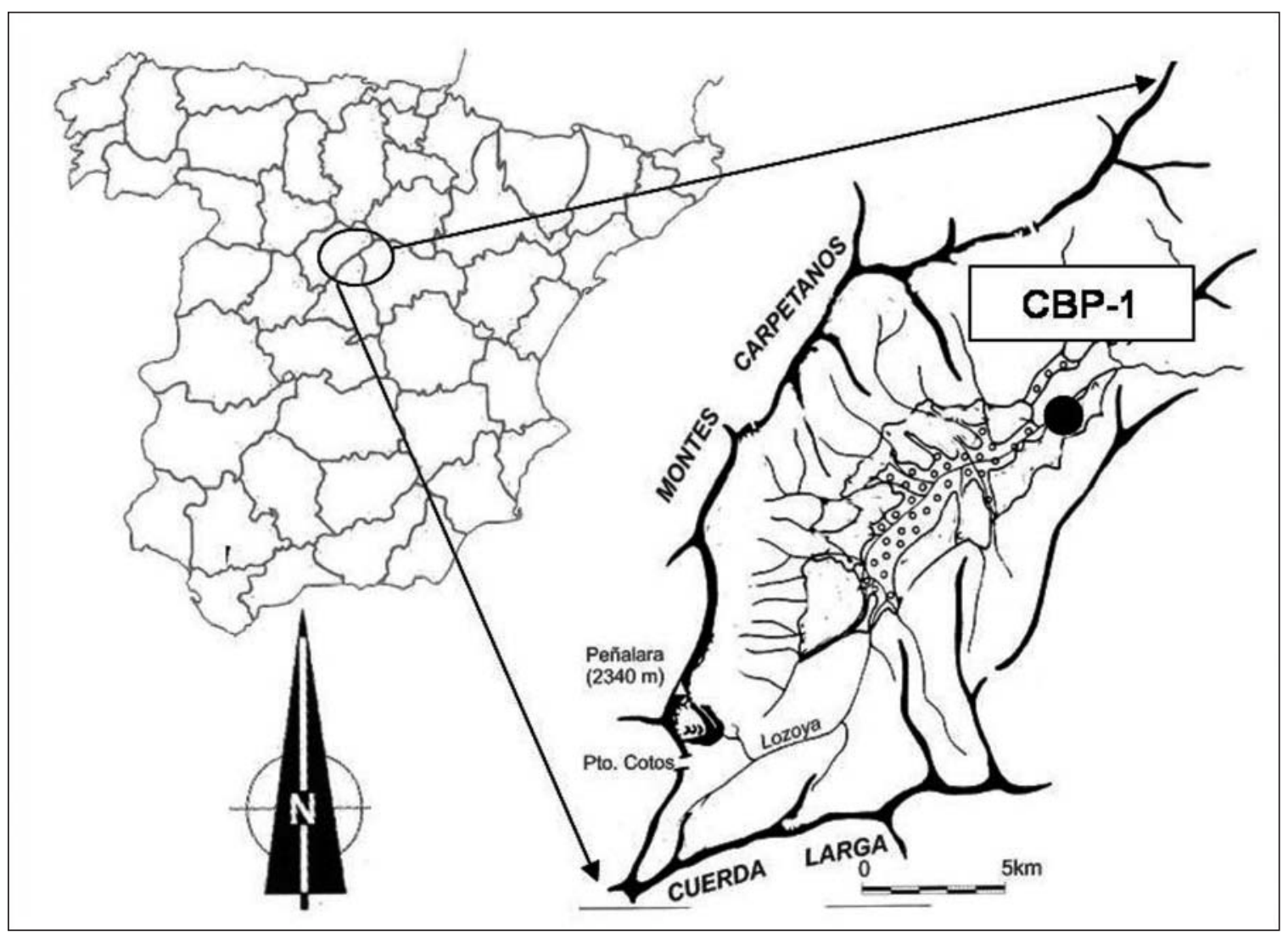

Figura 1. Situación geográfica de la secuencia CBP (Cueva de la Buena Pinta). Basado en Pedraza \& Carrasco (2005). Geographic situation of CBP sequence (Buena Pinta Cave. Found in Pedraza \& Carrasco (2005). 
treó, para su estudio palinológico, la secuencia holocénica, en un perfil de 1,80 $\mathrm{cm}$ de potencia, abierto en el sector norte de la cueva (CBP1). Esta secuencia sella la actual boca de entrada y reposa sobre el relleno sedimentario del Pleistoceno superior, que colmata parcialmente dicha cavidad. Desde el punto de vista litológico se diferencia una unidad inferior, entre los 180-65 cm, correspondiente a un depósito de gravas polimíctico, matriz soportado, embebido en una matriz arenosa, mal seleccionado, constituido por bloques de caliza y brechas con huesos, entre 40-50 cm de eje mayor acumulándose el resto de los tamaños entre 3-4 a $20 \mathrm{~cm}$. La superior, entre los $65-12 \mathrm{~cm}$, se apoya sobre una superficie de erosión y se trata de un depósito de gravas matriz soportado embebido en una arena de fina muy fina con clastos de $2-5 \mathrm{~cm}$. A techo se desarrolla un horizonte edáfico Ap con bioturbación; las dataciones radiométricas de ${ }^{14} \mathrm{C}$ sitúan, su desarrollo, con anterioridad a 5740-5600 cal BP.

Los datos obtenidos han puesto de manifiesto la evolución progresiva de un paisaje abierto, como respuesta a la instalación de unas condiciones climáticas de carácter cálido y seco. El bosque original, formado por Quercusp, Quercus-c, Fraxinus, Corylus y taxones riparios como Salix y Alnus, presenta en la base del diagrama polínico, los primeros síntomas de deterioro, dando paso a una fase de dominio de matorral, constituido por Juniperus y en menor medida Ericaceae y Rosaceae. El desarrollo del matorral va asociado a una disminución generalizada de los taxones arbóreos tanto de carácter local como regional (Pinus); el descenso de los taxones riparios va unido al aumento de los taxones acuáticos. La permanencia de las condiciones climáticas favorece el desarrollo de una nueva fase, en este caso dominada por el estrato herbáceo, debido a la expansión de Chenopodiaceae-Amaranthaceae, así como de Asteraceae tipo tubuliflorae y finalmente de Asteraceae liguliflorae definiendo tres fases a lo largo de las cuales quedan patentes pequeñas fluctuaciones en la tasa de humedad y en la ocupación del territorio. Estos hechos quedan reflejados en las variaciones cualitativas y cuantitativas de los taxones riparios y acuáticos y en las presencias de los taxones herbáceos nitrófilos antropozoógenos (Rumex y Plantago lanceolata) así como en los de posible interés económico (Apiaceae, Fabaceae, Boraginaceae, Caryophyllaceae y Brassicaceae). A ello se une el desarrollo de palinomorfos no polínicos indicadores de fenómenos erosivos posiblemente asociados a la deforestación del entorno (Glomus cf. fasciculatum, tipo 207); las presencias puntuales de los tipos no polínicos $3 \mathrm{~b}, 16$, 182 y Spirogyra, podrían estar relacionadas con las fluctuaciones detectadas en la tasa de humedad.

La secuencia culmina con una expansión del bosque regional, constituido por Pinus, cuya lluvia polínica enmascara la presencia de los taxones arbóreos de carácter local, e impide el desarrollo del estrato arbustivo; se detecta una mayor representación cualitativa de los taxones acuáticos, de los palinomorfos tipo 207 y tipo113 (indicador de condiciones coprófilas), así como un descenso en los taxones herbáceos de carácter nitrófilo.

\section{MATERIAL Y MÉTODOS}

Dada la aparente homogeneidad litológica del perfil, la toma de muestras se realizó, de modo continuo, en intervalos de $5 \mathrm{~cm}$. El tratamiento químico de las mismas, para aislar los granos de polen, se ajustó a los protocolos estándar, mediante el empleo de ácidos y álcalis, y el residuo fue sometido a técnicas de enriquecimiento, con licor denso de Thoulet. La lectura de las muestras se realizó sobre un volumen de muestra de $40 \mu \mathrm{m}$, llevando a cabo la contabilización tanto del contenido de polen como la de otros microfósiles no polínicos. Estos microfósiles no polínicos, identificados siguiendo la tipología numérica establecida por la escuela de B. van Geel de la Universidad de Amsterdam (Holanda) constituyen una herramienta adicional a la hora de interpretar las condiciones ambientales del entorno; de muchos de ellos aun se desconoce la afinidad ecológica o la interpretación es aún muy general, pero algunos son claros indicadores de presión pastoral (tipo 113), representativos de períodos secos (tipos 16 y 3b), períodos húmedos (tipo182 y Spirogyra) o de fases erosivas en el medio (tipo 207).

El perfil cuenta con tres dataciones radiométricas ${ }^{14} \mathrm{C}$ realizadas en el laboratorio Beta Analytic (Tabla 1), que ubican la formación de dicho relleno desde el 5.740-5.600 cal. BP a los $150 \mathrm{~cm}$, y los 1.940-1.800 BP de los $45 \mathrm{~cm}$, desarrollándose entre finales del Holoceno medio e inicios del Holoceno reciente

\section{RESULTADOS}

En la secuencia polínica del perfil PV-CBP1 (Fig. 2), se han identificado un total de 30 taxones de los cuales 9 son arbóreos, 6 arbustivos y 15 herbáceos, junto a 5 taxones acuáticos, además de 10 microfósiles no polínicos. En cuanto a la composición, destaca Pinus como elemento de carácter regional, frente a la vegetación local definida fundamentalmente por elementos de ribera (Alnus, Salix, Fraxinus y Ulmus) junto a taxones termófilos (Quercus ilex) y mesófilos (Corylus, Juglans y Quercus pyrenaica) que, en principio, definen unas condiciones de carácter templado mediterráneo. El grupo arbustivo está constituido principalmente por Juniperus y en menor medida Cistaceae, Calluna, Ericaceae y Rosaceae. En el conjunto herbáceo, el hecho más destacable es la escasa diversidad que presenta y el dominio por parte de Asteraceae tubuliflorae y liguliflorae y Chenopodiaceae-Amaranthaceae, el resto de los elementos se distribuye a modo de presencias a lo largo del perfil. En la secuencia destaca 


\begin{tabular}{|l|c|c|c|c|c|}
\hline MUESTRA & Lab. No. & $\begin{array}{c}\text { Prof. } \\
(\mathrm{cm})\end{array}$ & Edad convencional C 14 (BP) & $\begin{array}{c}\text { Edad calibrada } \\
\text { cal. AD/BC }(2 \sigma)\end{array}$ & $\begin{array}{c}\text { Edad calibrada } \\
\text { cal BP }(2 \sigma)\end{array}$ \\
\hline PinillaCBPN1 & Beta-199880 & 45 & $1.920 \pm 40$ & $10-150 \mathrm{AD}$ & $1.940-1.800$ \\
\hline PinillaCBPN2 & Beta-199881 & 75 & $4.010 \pm 40$ & $2.600-2.460 \mathrm{BC}$ & $4.560-4.410$ \\
\hline PinillaCBPN3 & Beta-199882 & 150 & $4.940 \pm 40$ & $3.790-3.650 \mathrm{BC}$ & $5.740-5.600$ \\
\hline
\end{tabular}

Tabla 1. Dataciones ${ }^{14} \mathrm{C}$.

Radiocarbon dating ${ }^{14} \mathrm{C}$.

la presencia continua de los elementos acuáticos, lo que unido a la presencia del bosque de ribera define un grado de humedad, al menos de disponibilidad de agua canalizada importante. Todo ello indica la existencia en la zona de unas condiciones de mediterraneidad con cierto grado de humedad.

En cuanto al comportamiento de la vegetación, de un modo general se observa una tendencia hacia la instalación de paisajes abiertos, marcada por el fuerte retroceso de la masa forestal, asociado a una pérdida de la diversidad. Destaca la presencia constante de los elementos de ribera y acuáticos, la escasa representación del pinar y la pérdida de los taxones arbóreos de carácter templado y mediterráneo, junto al desarrollo de Juniperus y el aumento progresivo de los taxones herbáceos Asteraceae tubuliflorae y liguliflorae. De la distribución de los componentes a lo largo del perfil, se detectan una sucesión de cambios muy significativos que permiten diferenciar tres zonas polínicas.

\section{Zona-I}

Se define para el intervalo comprendido entre 125-180 $\mathrm{cm}$, desarrollado alrededor de los 5.740-5.600 cal BP; está caracterizada por presentar el mayor desarrollo del bosque constituido por Quercus perenne (Quercus-p) y Corylus, junto a Fraxinus y Quercus caduco (Quercus-c) y en menor proporción Pinus, asociado a un pobre pero variado componente herbáceo, en cuyo seno están presentes herbáceas tipo Asteraceae tubuliflorae, Chenopodiaceae-Amaranthaceae y bajos porcentajes de Poaceae. Los taxones acuáticos (Cyperaceae y Ranunculaceae) están bien representados frente a una modesta representación de los taxones de ribera. Dentro de los microfósiles no polínicos destaca la presencia del tipo 207 (asociado a procesos erosivos). En esta zona, el comportamiento de los taxones define dos pulsaciones:

Zona Ia: $(180-147 \mathrm{~cm})$ representa los eventos acaecidos con anterioridad a los 5.740-5.600 cal BP, momento en que la vegetación está dominado por un bosque denso (70\% del total de la vegetación) de tipo mixto con Fraxinus, Quercus-p y Quercus-c, acompañados por Corylus, Salix y Ulmus y finalmente, Pinus de carácter regional, que muestra los primeros síntomas de retroceso. Bajo este bosque relativamente denso, los estratos arbustivo y herbáceo están poco representados.

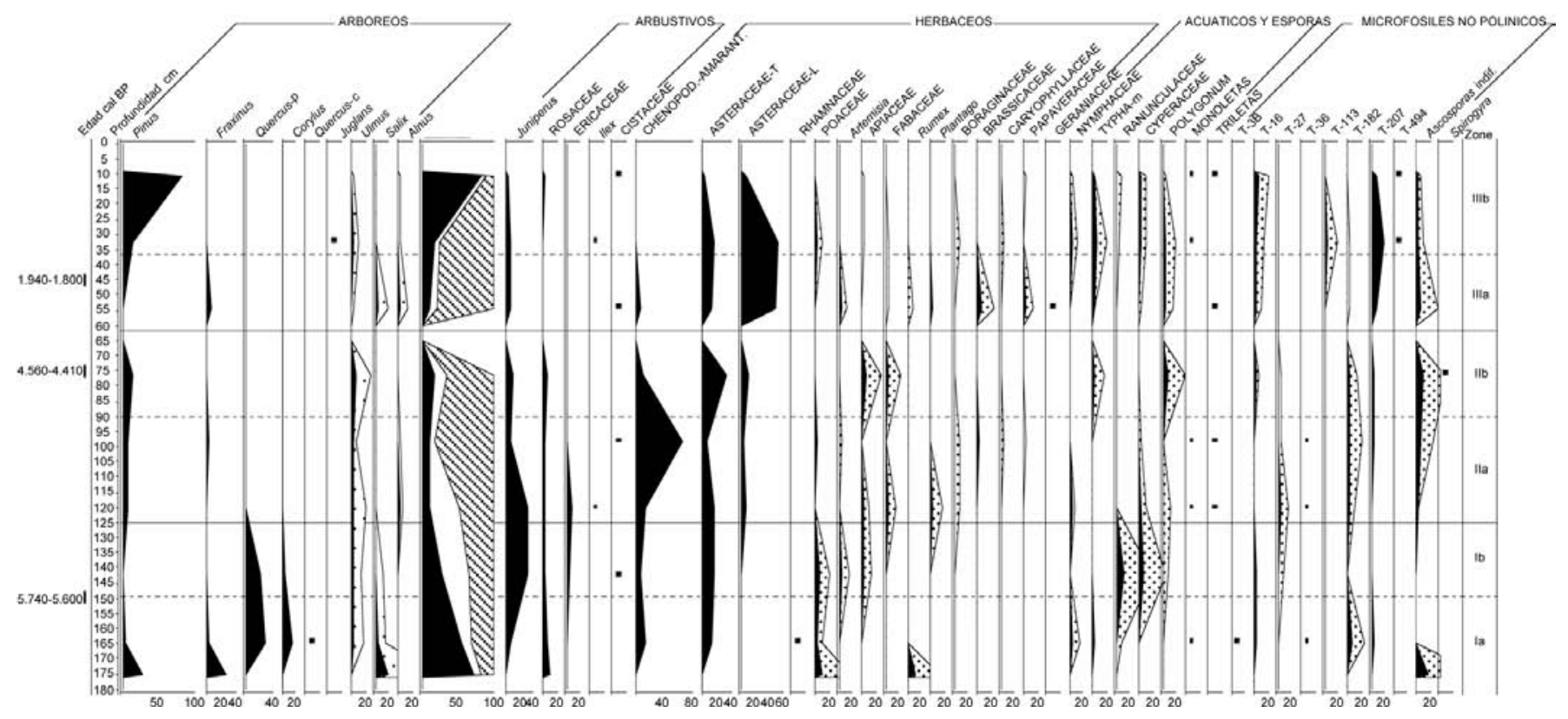

Figura 2. Diagrama polínico CBP. Pollen diagram CBP. 
Zona Ib: (147-125cm) constituye una fase de dominio arbustivo, como consecuencia del desarrollo de un matorral fundamentalmente de Juniperus y en menor medida de Ericaceae, como respuesta al deterioro progresivo del bosque, que llega a alcanzar valores de tan sólo el 15\%. Este proceso va asociado a la pérdida de Fraxinus, Quercus-c y Corylus y al descenso de Quercus-p, de los taxones de ribera y de Pinus. El grupo herbáceo todavía es, porcentualmente, poco significativo. Se observa un aumento de los taxones acuáticos (Cyperaceae, Ranunculaceae y Polygonaceae), probablemente asociado con el descenso de los taxones de ribera, como consecuencia de la pérdida de disponibilidad de agua encauzada.

\section{Zona-II}

Abarca el intervalo de 125 a $63 \mathrm{~cm}$ y representa la primera etapa de dominio herbáceo, debido fundamentalmente a la expansión, primero de Chenopodiaceae-Amaranthaceae, a continuación de Asteraceae tubuliflorae y finalmente de Asteraceae liguliflorae. Estas sucesivas sustituciones, van acompañadas de cambios menores tanto en el grupo arbóreo, como en el arbustivo y herbáceo, definiendo dos pulsaciones, desarrolladas alrededor de 4.560$4.410 \mathrm{cal} \mathrm{BP}$.

Zona IIa: $(125-90 \mathrm{~cm})$ representa los eventos acaecidos con anterioridad a los 4.560-4.410 cal BP. Corresponde a la expansión de Chenopodiaceae-Amaranthaceae, y es coincidente con el retroceso de Juniperus, y una pequeña expansión de Ericaceae, Cistaceae e Ilex. Pinus experimenta una ligera mejoría y se detecta una pequeña reintroducción de Fraxinus, alcanzando valores similares a los de Quercus-p. Junto a Ulmus se detecta la presencia de Alnus. En el grupo herbáceo destacan además los valores alcanzados por Poaceae y Plantago.

Zona IIb: $(90-63 \mathrm{~cm})$ representa la expansión de Asteraceae tubuliflorae, junto a Apiaceae y Fabaceae; se detecta un ligero aumento del bosque debido al incremento de Pinus y Ulmus; mientras que no están presentes otros taxones de ribera. Dentro de los elementos acuáticos sólo Typha y Polygonaceae están representados, no se detectan tampoco esporas y se produce un incremento de los microfósiles no polínicos tipo 207 y tipo 16 y tipo 182 .

\section{Zona-III}

Abarca el intervalo de 63 a $10 \mathrm{~cm}$ y representa la segunda etapa de dominio herbáceo, en este caso debido fundamentalmente a la expansión de Asteraceae tubuliflorae y finalmente de Asteraceae liguliflorae, dando paso una fase de expansión del bosque. Esta definida por dos pulsaciones, desarrolladas alrededor de 1940-1800 cal BP.

Zona IIIa: $(63-37 \mathrm{~cm})$, esta unidad se desarrolla tras un proceso erosivo que afecta a la unidad II b y está datada con anterioridad a 1.940-1.800 cal. BP. Corresponde a una fase de expansión de Asteraceae liguliflorae, junto con Artemisia, Fabaceae, Rumex, Plantago y Brassica- ceae, unido a la expansión del tipo 207 y en menor medida del tipo 16 y Cistaceae. Los taxones arbóreos como Alnus, Salix y Ulmus están poco representados, al igual que, Quercus-p y Fraxinus; a ello se une la una importante reducción del pinar.

Zona IIIb: comprende el intervalo de $37-10 \mathrm{~cm}$ y se define como una fase de expansión del bosque, debido al desarrollo de Pinus, otros taxones arbóreos como Alnus, Salix y Ulmus e incluso Juglans, se detectan a través de presencias. El estrato arbustivo es escaso y está constituido por Juniperus junto a presencias de Rosaceae, Ericaceae, Cistaceae e Ilex. El conjunto herbáceo aparece poco desarrollado, debido a la reducción porcentual de Chenopodiaceae-Amaranthaceae y de los dos tipos de Asteraceae, lo que parece favorecer un aumento de su diversidad. Aumentan los taxones acuáticos y los tipos 16, 207 y 494.

\section{DISCUSIÓN}

El inicio de la secuencia, con anterioridad a 5.740-5.600 cal BP muestra un paisaje dominado por un bosque relativamente denso constituido por melojos y avellanos en el que también se encontraban presentes encinas y fresnos, mientras que en las zonas montañosas limítrofes existirían bosques de pinos. Este paisaje es similar al encontrado en el depósito de Rascafría para este periodo (Franco, 1995) y Peñalara (Ruiz Zapata et al., 1996), que estaría de acuerdo con la existencia de un periodo de mejoría climática. Lamentablemente en nuestro depósito no hemos obtenido información del "óptimo climático del Holoceno", durante el cual se dio un espectacular crecimiento de los bosques en la región (Andrade et al., 1997), tan sólo tenemos información de los últimos momentos del mismo en el que existió un cierto atemperamiento climático. A ello se une un cierto grado de actividad antrópica sobre el territorio evidenciada por la presencia de taxones antropozoógenos lo que en cierta mediada enmascara los efectos del clima sobre la vegetación.

Tras esta etapa inicial se produce un drástico retroceso del bosque; inicialmente estas formaciones boscosas fueron sustituidas por un matorral en el que se encontraba bien desarrollado Juniperus junto con ericaceas, rosaceas y cistaceas. Estos datos estarían de acuerdo con el empeoramiento de las condiciones climáticas que tuvieron lugar entre 5.0002.500 BP (Andrade et al., 1997), con una tendencia al enfriamiento. Es un periodo muy inestable con momentos de precipitaciones intensas, durante las cuales a nivel regional se dieron ligeras expansiones de Betula e Ilex (Andrade et al., 1997) mientras que en nuestra secuencia sólo se observa un pequeño incremento de Ilex y de los taxones acuáticos. No hay que olvidar, que en el tránsito de la zona IIb a la IIIa se produce una erosión que hace que parte de la información no haya quedado reflejada en nuestro diagrama. La inestabilidad durante este periodo produjo sequías muy severas. Estas fuertes sequías quedan constatadas en nues- 
tra secuencia por el importante desarrollo experimentado por Chenopodiaceae-Amaranthaceae, junto con el retroceso de los taxones acuáticos (IIa). El final de este periodo se caracteriza por una fuerte deforestación como consecuencia tanto de variaciones en los parámetros climáticos como al incremento de la acción humana sobre el medio. Nuevamente en todos los registros del área (Andrade et al., 1997) muestran la misma dinámica con un retroceso del bosque, siendo Pinus el taxon más afectado fundamentalmente en los depósitos de montaña, alcanzando en nuestra secuencia los mínimos valores (IIIa), simultáneamente se produce un espectacular incremento de Asteraceae liguliflorae.

Seguidamente, tiene lugar una fase con precipitaciones ligeramente superiores que ha sido detectada en la mayoría de los registros de la zona, en torno a los 2.500-2.000 $\mathrm{BP}$, y que en este registro se constata a través de un ligero incremento de los taxones acuáticos (final IIIa).

Con posterioridad se produce una intensificación de la actividad antrópica que afecta incluso al matorral, el cual aparece muy mermado en nuestra secuencia (inicio IIIb), frente al importante desarrollo de las herbáceas en este momento pero con una tendencia al retroceso.

Tras esta etapa se produce una rápida recuperación del pinar regional debido tal vez a las medidas de intervención (Plan Provisional de Aprovechamiento Forestal 1873-74) o quizá a la regeneración natural del pinar tras regular su explotación.

Esta información pone de manifiesto la existencia de una vegetación para finales del Holoceno medio de un bosque bien formado y mixto en vías de degradación, que da paso a una fase de carácter más seco a lo que habría que añadir la actividad antrópica en el valle que se va haciendo cada vez más intensa con una importante pérdida de la masa forestal, si bien desde finales del siglo XIX se inicia una nueva recuperación de la misma.

La discordancia erosiva entre IIb y IIIa podría ser la causa que explicara la falta de información en nuestro depósito, del Episodio Cálido Romano definido por recuperación de carácter térmico.

\section{BIBLIOGRAFÍA}

Alférez, F. 1985. Dos molares humanos procedentes del yacimiento del Pleistoceno Medio de Pinilla del Valle (Madrid). Trabajos de Antropología, 19 (4), 303.

Alférez, F. \& Molero, G. 1982. Descubrimiento de un fósil humano (Riss-Wurm) en Pinilla del Valle (Madrid). Résumés des Comunications du I Congrés International de
Paleontologie Humane. Nice. Section IV : Homo sapiens neandertalensis/Neandertaliens et Neandertaloides, 15-32.

Alférez, F. \& Roldán,B. 1992. Un molar humano Anteneardental con patología traumática procedente del yacimiento Cuaternario de Pinilla del Valle (Madrid). Munibe, 8, 183-188.

Andrade, A., Arnanz, A.M., Dorado, M., Gil, M.J., Franco, F., López, P., López, J.A., Macías, R., Pedraza, J., Ruiz, B., \& Uzquiano, P. 1997. El Paisaje Vegetal de la Comunidad de Madrid durante el Holoceno final. Arqueología, Paleontología y Etnografía, Monográfico, 5, Comunidad de Madrid, Madrid, $201 \mathrm{pp}$.

Baquedano, E., Bermúdez de Castro, J.M., Pérez-González, A., Márquez, B., Laplana, C., Baena, J., Bajo, S., Espinosa, J.A., Gómez, J., Huguet, R., Made, J. Van Der, Maldonado, E., Martínez, C., Mateos, A., Molero, G., Nicolás, E., Panera, J., Rodríguez, J., Romero, B., Sarmiento, S. \& Sevilla, P. 2004. Excavaciones arqueológicas en Pinilla del Valle (Madrid). IV Congreso de Arqueología Peninsular, 2 pp.

Fernández, F. 1988. Estudio florístico y fitosociológico del Valle del Paular (Madrid). Tesis Doctoral, Universidad Complutense de Madrid, 759 pp. (Inédita).

Franco Múgica, F. 1995. Estudio palinológico de turberas holocenas en el Sistema Central: reconstrucción paisajística y acción antrópica. Tesis Doctoral, Universidad Autónoma de Madrid, 358 pp. (Inédita).

Márquez, B., Baquedano, E., Bermúdez de Castro, J.M. \& Pérez-González, A. 2004. Yacimientos de Pinilla del Valle (Madrid). XX Jornadas de la Sociedad Española de Paleontología. Guía de la excursión. Alcalá de Henares, $12 \mathrm{pp}$.

Pedraza, J. \& Carrasco, R.M. 2005. El glaciarismo pleistoceno del Sistema Central. Enseñanza de las Ciencias de la Tierra; monográfico: Los Glaciares, 13, 278-288

Peinado Lorca, M. \& Rivas-Martínez, S. 1987. La Vegetación de España. Servicio de Publicaciones de la Universidad de Alcalá, Alcalá de Henares, 544 pp.

Ruiz Zapata, M.B. 2004. Análisis polínico del yacimiento de Pinilla del Valle (Madrid): sector Camino. XX Jornadas de la Sociedad Española de Paleontología. Guía de la excursión. Alcalá de Henares, 2 pp.

Ruiz Zapata, M.B., Gil García, M.J. \& Dorado Valiño, M. 1996. Climatic changes in the Spanish Central South during the last 3000 BP based on pollen analysis. In: Diachronic climatic impacts on Water Resources with Emphasis on Mediterranean Region (Ed. A.N. Angelakis \& A.S. Issar). Springer, Berlín, 36, 9-23.

Manuscrito recibido: 15 de noviembre, 2006 Manuscrito aceptado: 17 de abril, 2007 